\title{
THE EPIDEMIOLOGY OF POST-NEONATAL HYPERNATRAEMIA AND THE DIFFERENTIATION OF SALT POISONING (SP) AND OSMOSTAT DYSFUNCTION FROM COMMON CAUSES
}

\author{
N. McIntosh ${ }^{1}$, S. Forman ${ }^{1}$, H. Huang ${ }^{1}$, T. Marshall ${ }^{1}$, P. Crofton ${ }^{2}$ \\ ${ }^{1}$ Child Life and Health, University of Edinburgh, ${ }^{2}$ Paediatric Biochemistry, Royal Hospital for Sick Children, \\ Edinburgh, UK
}

Aim: To investigate the epidemiology of postneonatal hospital admission hypernatraemia in children in Lothian region, Scotland

Population denominator: 1,239,360 children $<17$ years over 10 years

Method: The reasons for admission of children with plasma sodium values $=>150 \mathrm{mmol} / 1$ were evaluated over a 10 year period.

Results: 42 cases were found: 21 from gastroenteritis, 13 from systemic infections, 1 from diabetes mellitus, 3 in short bowel syndrome and home parenteral nutrition, 2 from diabetes insipidus, 1 from failure of breast feeding (readmission) and 1 from salt poisoning (SP) (11 admissions starting at 5.2y). The incidence of hypernatraemia in children $<17$ years of age is $1: 28,822$.

During this period a case of osmoreceptor dysfunction (OD) had 5 admissions. A comparison of the plasma biochemistry was made:

Dehydration; OD; SP;

Patient episodes - 33; 5; 11

Age, years - 0.8; 8.6; 7.3

Sodium, mmol/L - 153 (150 - 175;) 152 (152 - 155); 156 (151 - 169)

Urea, mmol/L - 11.7 (2.7 - 48.6); 6.2 (4.0 - 6.4); 3.2 (1.9 - 5.4)

Creatinine, mmol/L 78 (46 - 253); 68 (60 - 82); 58 (52 - 83)

Discussion: Many pathologies causing dehydration lead to hypernatraemia but SP and OD are difficult to rule out. Any repeat admission with hypernatraemia requires a full investigation to exclude significant neuropathology and the rare case of SP. Paired urine and plasma samples for electrolytes, urea, and creatinine taken immediately on admission will usually allow these conditions to be ruled out. 